\title{
Simulation Study of AIN Spacer Layer Thickness on AlGaN/GaN HEMT
}

\author{
Niraj Man Shrestha ${ }^{1}$, Yuen Yee Wang ${ }^{1}$, Yiming $\mathrm{Li}^{2, *}$, and *E.Y. Chang ${ }^{1}$ \\ ${ }^{1}$ Compound Semiconductor Device Laboratory, Department of Material Science and Engineering \\ ${ }^{2}$ Parallel and Scientific Computing Laboratory, Department of Electrical and Computer Engineering \\ National Chiao Tung University, 1001 University Road, Hsinchu 300, Taiwan \\ *edc@mail.nctu.edu.tw
}

\begin{abstract}
High electron mobility transistor (HEMT)Two-dimensional electron gas (2DEG) formed at AlGaN/GaN interface is a critical part to tune the characteristic of AlGaN/GaN HEMT devices. Introduction of AlN spacer layer in between $A l G a N$ and GaN layer is one of the way to improve $2 D E G$ density, mobility, and drain current. Carrier concentration, mobility and conduction band offset for different spacer layer thickness was simulated by using Silvaco simulation tool. Our device simulations showed that carrier concentration, mobility are enhance on introduction of AlN spacer layer in HEMT. In addition, carrier properties of HEMT also depend on thickness of spacer layer. Our simulation showed that the mobility of $2 D E G$ attains its maximum value at the $0.5 \mathrm{~nm}$ thick AlN layer but carrier concentration increases with spacer thickness. Finally, drain current increases with increasing spacer layer thickness and reach maximum value at $1.2 \mathrm{~nm}$ thick spacer layer.
\end{abstract}

Keywords: HEMT, Spacer layer, mobility, carrier concentration, scattering, simulation

\section{INTRODUCTION}

$\mathrm{AlGaN} / \mathrm{GaN}$ high electron mobility transistor (HEMT) have been the cardinal subject of many recent investigations because of their material properties such as large band gap $(3.45 \mathrm{eV})$, high breakdown field $(3 \mathrm{MeV})$, high saturation velocity $\left(2.2 \times 10^{7} \mathrm{~cm} / \mathrm{sec}\right)[1] \mathrm{etc}$. These crucial material properties lead the device has high potential for use in high-temperature, high-power devices. One of the most unusual features of these HEMT is that very high 2 DEG densities $\left(10^{13} \mathrm{~cm}^{-2}\right)$ can be found in even nominally or undoped heterostructures [24]. Usually, for high $2 \mathrm{DEG}$ carrier density at the $\mathrm{AlGaN} / \mathrm{GaN}$ interface, higher $\mathrm{Al}$ mole fraction in the AlGaN barrier layer can be used [5]. However, Low quality $\mathrm{AlGaN}$ barrier layer for high $\mathrm{Al}$ content on $\mathrm{GaN}$ and alloy disorder scattering lead drop of mobility [6].

Therefore, the product of mobility and carrier concentration improvement is limited which is very crucial for high power density of device. To improve the electrical properties of HEMTs, Shen et al. had reported the AlGaN/AlN/GaN Microwave HEMT [7]. The AlN spacerlayer in between AlGaN and $\mathrm{GaN}$ layer eliminates the strain of the $\mathrm{AlGaN}$ layer [8] and the film quality of the AlGaN layer has been improved. An additional thin AlN spacer between $\mathrm{AlGaN}$ and $\mathrm{GaN}$ improves the mobility at low Temperatures, where the thickness of AlN is an important parameter for the mobility in AlN/ $\mathrm{GaN}$ heterostructures [9-11]. In this study, the electron transport for different AlN spacer thickness is simulated by using Silvaco simulation tool. In addition, Ohmic electrode is recessed in order to reduce the ohmic resistance.

\section{SIMULATION METHEDOLOGY}

Fig. 1 shows the schematic of explored device; the device characteristic was simulated by solving a set of quantum mechanically corrected transport equations.

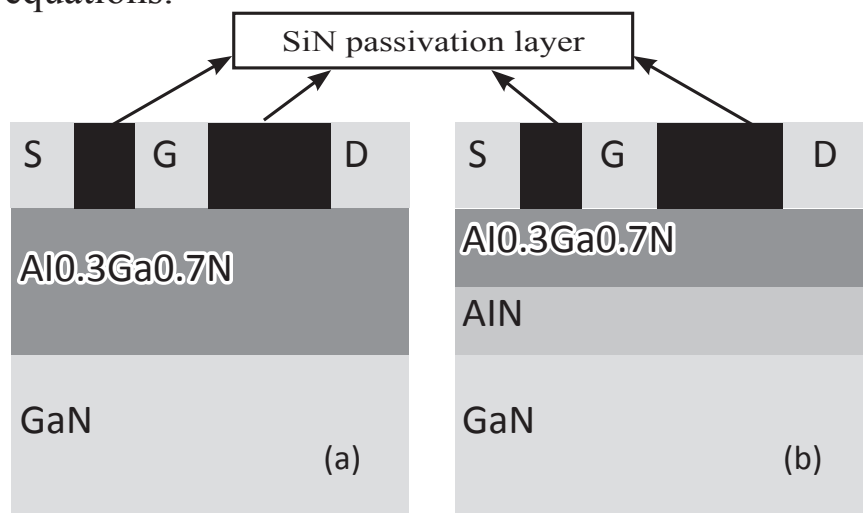

Fig.1. the simulated structure of HEMTs, where the plots (a) and (b) are without and with the AlN spacer Parameters used in this work are in table number 1. 


\begin{tabular}{|l|l|}
\hline Work function of gate & $5.18 \mathrm{eV}$ \\
\hline Gate width $(\mathrm{w})$ & $100 \mu \mathrm{m}$ \\
\hline Source Gate space $\left(\mathrm{L}_{\mathrm{sg}}\right)$ & $1.5 \mu \mathrm{m}$ \\
\hline Drain Gate space $\left(\mathrm{L}_{\mathrm{gd}}\right)$ & $4 \mu \mathrm{m}$ \\
\hline Al fraction $(\mathrm{x})$ & 0.30 \\
\hline AlGaN thickness $\left(\mathrm{t}_{\text {alGaN }}\right)$ & $25 \mathrm{~nm}$ \\
\hline GaN thickness $\left(\mathrm{t}_{\mathrm{GaN}}\right)$ & $2 \mu \mathrm{m}$ \\
\hline gate length $\left(\mathrm{L}_{\mathrm{g}}\right)$ & $1 \mu \mathrm{m}$ \\
\hline
\end{tabular}

In this device gate length, Source gate distance and gate drain distance are $1 \mu \mathrm{m}, 1.5 \mu \mathrm{m}$ and $4 \mu \mathrm{m}$ respectively. $\mathrm{AlGaN}$ and $\mathrm{GaN}$ layers thickness are $25 \mathrm{~nm}$ and $2 \mu \mathrm{m}$ respectively. Al composition fraction of $\mathrm{AlGaN}$ layer is $30 \%$. Work function of Gate metal is $5.18 \mathrm{eV}$.Owing to n-type uniform doping concentration at Source and Drain regions are $1 \times 10^{18} \mathrm{~cm}^{-3}$, source and drain contacts become ohmic. To obtain numerical results for a device, the device is simulated by solving classical transport equations using the commercial tool ATLAS [12]. To adopt the current relationship Drift diffusion model [12] is used.

$$
\vec{J}=q D_{n} \nabla n-q n \mu_{n} \nabla \psi-\mu_{n} n\left\{k T_{L} \nabla\left(\ln n_{i e}\right)\right\} \ldots
$$

Where $\mathrm{q}$ is charge, $\mathrm{D}$ is diffusion coefficient, is electrostatic potential, $\mathrm{T}_{\mathrm{L}}$ is lattice temperature, $\mathrm{n}_{\mathrm{ie}}$ is intrinsic concentration

To study the effect of electric field on transport properties, low field and nitride specific field dependent mobility model are used in the simulation. Low field mobility is expressed [12] as

$$
\frac{1}{\mu}=\frac{a \cdot N}{b}\left(\frac{T_{L}}{c}\right)^{-\frac{3}{2}} \ln \left[1+3\left(\frac{T_{L}}{c}\right)^{2}\left(\frac{N}{b}\right)^{-\frac{3}{2}}\right]+d\left(\frac{T_{L}}{c}\right)^{\frac{3}{2}}+\frac{x}{\exp \left(\frac{y}{T_{L}}\right)_{-1}}
$$

Where $\mu$ is mobility depends on doping (N) and lattice temperature $\left(T_{L}\right)$ and $a, b, c, d, x$ and $y$ are user specifiable parameters on the mobility statements.

The high field dependent mobility model [12] is given by

$$
\mu=\frac{\mu_{0}(T, N)+v^{s a t} \frac{E^{n 1-1}}{E_{C}^{n 1}}}{1+a\left(\frac{E}{E_{C}}\right)^{n_{2}}+\left(\frac{E}{E_{C}}\right)^{n 1}} \ldots \ldots . .
$$

Where $\mu$ is low filed mobility model, $\mathrm{E}$ is electric field and $\mathrm{v}^{\mathrm{sat}}, \mathrm{E}_{\mathrm{c}}, \mathrm{a}, \mathrm{n}_{1}$ and $\mathrm{n}_{2}$ are model parameters. Polarization model is used to activate polarization .Newton method is used to solve the numerical problems. Tony plot was used to see the output results.

\section{RESULT AND DISCUSSION}

Mobility and carrier concentrations are calculated for both the conventional HEMT and the HEMT which is with the AIN spacer layer between the layers AlGaN and GaN. Fig. 2 shows that improved electron concentration on inserting AIN spacer layer, which is mainly due to the increase in the quantum well depth, as shown in Fig. 3.

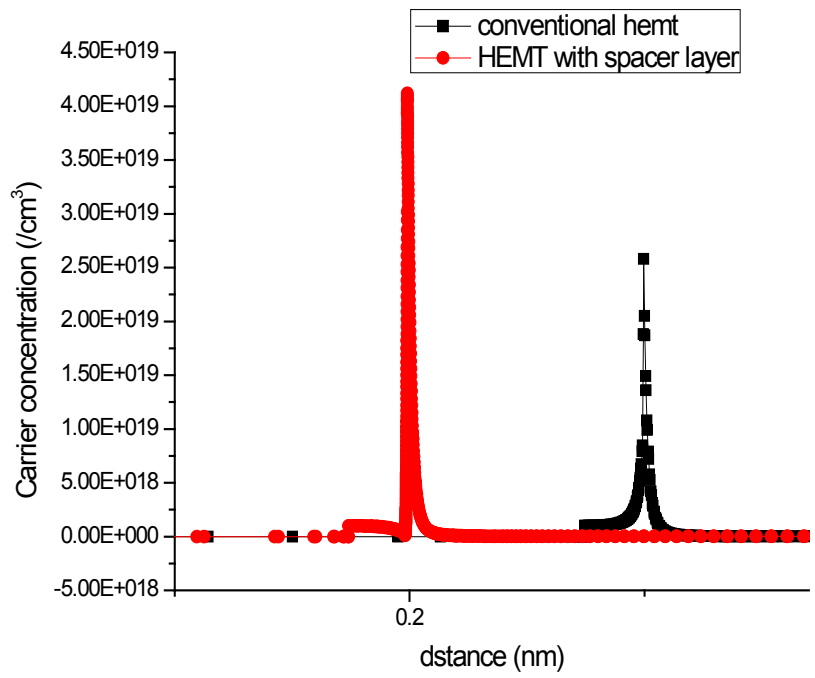

Fig. 2 The electron concentration for the device with and without AlN spacer layers.

Not shown here, the carrier mobility is also increased significantly. Due to the increase in quantum well depth, the scattering is lowered. In addition, alloy scattering is lowered because binary compound such as AlN has less alloy scattering in comparison to ternary compound [13]. As a result, the mobility is also increased, and, finally, the current is increased. 


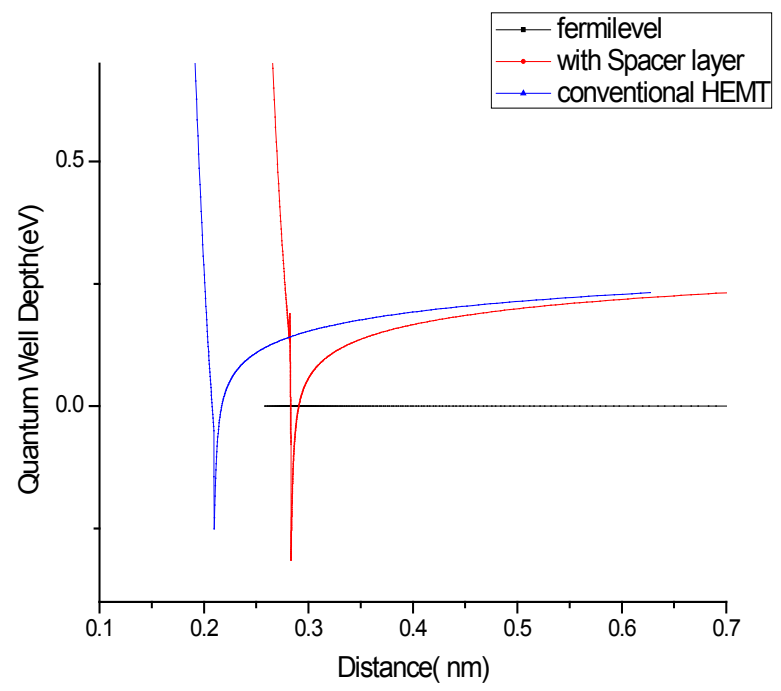

Fig. 3 The calculated quantum well depth for the device with and without AlN spacer layers.

We further explore the effect of AlN spacer layer thickness on the device characteristics. Quantum well depth and electron concentration are increased with AlN spacer layer thickness as shown Figs. 4(a) and 4(b). However, the electron mobility increases first, where the maximum appears at $0.5 \mathrm{~nm}$ AlN layer, and reduces on further increasing AlN layer, as shown in Fig. 5.

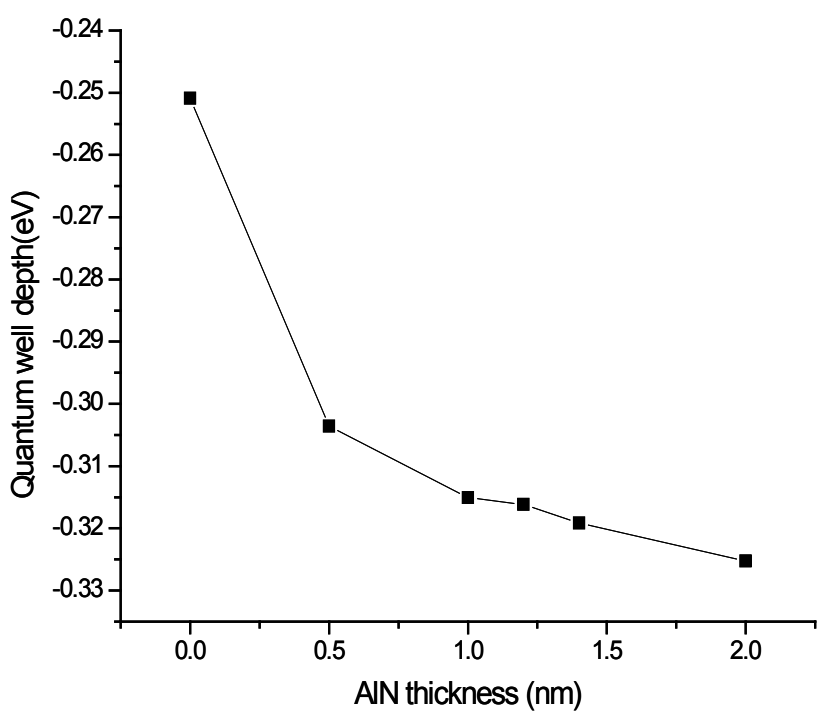

(a)

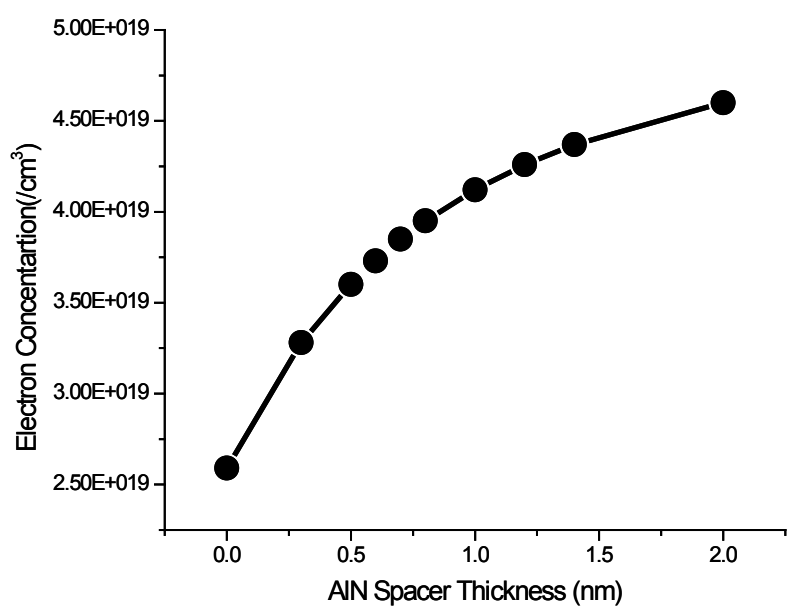

(b)

Fig. 4 (a) The quantum well depth and (b) the electron concentration with the AlN thickness.

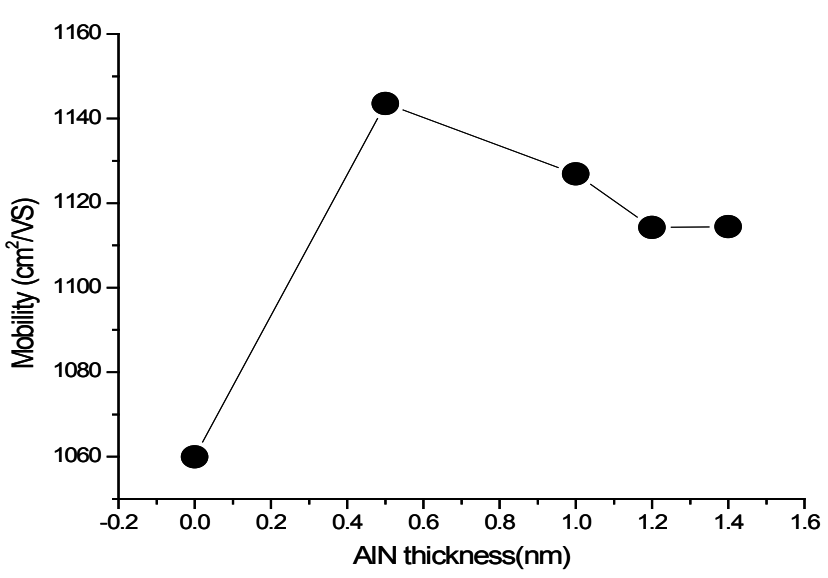

Fig.5 The mobility versus the AlN thickness.

This is owing to the Coulomb scattering between 2 DEG carriers when very thick spacer layer is used. As AlN spacer thickness is up to $0.5 \mathrm{~nm}$ Coulomb force between ions and with lattice is lower, which reduce the scattering and mobility is increased on increasing. However, as the thickness further increase beyond $0.5 \mathrm{~nm}$ width of quantum well is small enough and coulomb scattering between carriers become dominant. As a result, mobility is increased. 


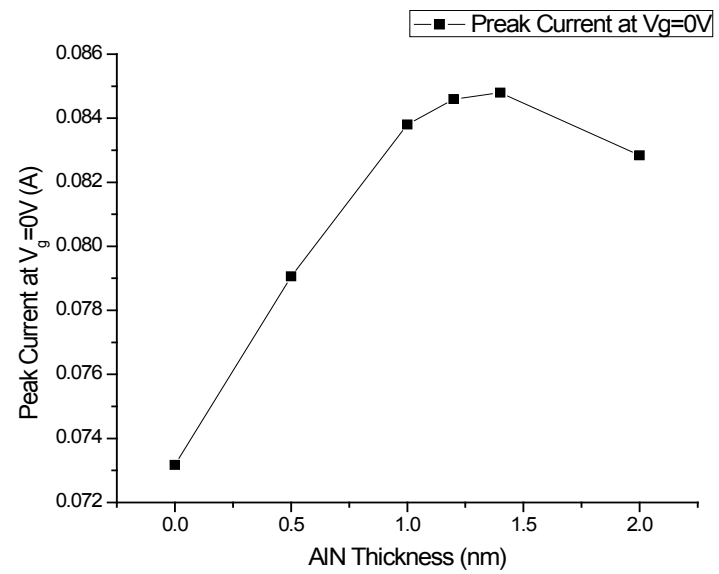

Fig.6. The simulated current for device with different AlN thicknesses

Fig. 6 shows the simulated drain current at the zero gate voltage. Current depend on carrier concentration and mobility. Up to AIN spacer layer thickness $1.2 \mathrm{~nm}$ carrier concentration increase play dominating role in current and current increase. Beyond $1.2 \mathrm{~nm}$ thickness decrease in mobility play dominating role on current. As a result, current is maximum at $1.2 \mathrm{~nm}$ and decrease on further increase of thickness.

\section{CONCLUSION}

The findings of the different thickness of AIN spacer layer conclude that electron concentration increases with the increases of AIN thickness. The device's mobility reaches the maximum at $0.5-\mathrm{nm}$ thick AIN layer and the drain current's maximum at $1.2 \mathrm{~nm}$ thick spacer.

\section{REFERENCES}

[1] MaxN.Yoder,"Measurementofpiezoelectrically induced charge in GaN/AlGaN heterostructure field-effect transistors" ieee transsection on electron device vol43,no.10,1996

[2] I. Smorchkova, C.R. Elsass, J.P. Ibbetson et al., "Measurement of piezoelectrically induced charge in GaN/AlGaN heterostructure fieldeffect transistors" J. Appl. Phys. 86, 4520 (1999)
[3] R. Gaska, J. W. Yang, A. Osinski et al., Electron transport in $\mathrm{AlGaN}-\mathrm{GaN}$ heterostructures grown on $6 \mathrm{H}-\mathrm{SiC}$ substrates Appl. Phys. Lett. 72, 707 (1998).

[4] E. T. Yu, G. J. Sullivan, P. M. Asbeck et al., "Measurement of piezoelectrically induced charge in GaN/AlGaN heterostructure fieldeffect Transistors”, Appl. Phys. Lett. 71, 2794 (1997).

[5] O. Ambacher, B. Foutz, J. Smart, J.R. Shealy, N.G. Weimann, K. Chu, M. Murphy, A.J. Sierakowski, W.J. Schaff, L.F. Eastman,R. Dimitrov, A. Mitchell, M. Strtzmann, J. Appl. Phys. 87 (2000) 334.

[6] G. Parish, S. Keller, S. P. Denbaars, and U. K. Mishra, "SIMS investigations into the effect of growth conditions on residual impurity and silicon incorporation in GaN and $\mathrm{Al} \mathrm{Ga} \mathrm{N}$, " J. Elect. Mater., vol. 29, no. 1,pp. 15-20, 2001.

[7] L. Shen, S. Heikman, B. Moran, R. Coffie, N.Q. Zhang, D. Buttari, I.P. Smorchkova, S. Keller, S.P. Denbaars, U.K. Mishra, IEEE Electron. Device Lett. 22 (10) (2001) 457.

[9] I. P. Smorchkova et al., "AlN/GaN and .Al,Ga./ AlN/GaN two-dimensional electron gas structures grown by plasma-assisted molecularbeam epitaxy” J. Appl. Phys. 90, 5196 (2001).

[10] Y. Cao et al., "High-mobility window for twodimensional electron gases at ultrathin AlN/ GaN heterojunctions" Appl. Phys. Lett. 90, 182112 (2007).

[11] M. Miyoshi, A. Imanishi, T. Egawa et al., "DC Characteristics in High-Quality AlGaN/ AlN/GaN High-Electron-Mobility Transistors Grown on AlN/Sapphire Templates" Jpn. J. Appl. Phys. 44, 6490 (2005).

[12] ATLAS manual

[13] D. Jena, I. Smorchkova, A. C. Gossard et. Al., phys. stat. sol. (b) 228, 617 (2001). 